\title{
Selection of Sampling Interval and Size of Random Sample for Radar Detection in the Moments Space
}

\author{
Camilo Guillén”, Giorgia Rodríguez¹, Nelson Chávez \\ 1 Department of Telecommunication and Telematics, Faculty of Telecommunication and Electronic, Universidad Tecnológica de \\ La Habana "José Antonio Echeverría", CUJAE, Calle 114 No. 11901 e/ Ciclovía y Rotonda, 19390 Marianao, Havana, Cuba \\ *Corresponding author, e-mail: camilo.gs@tele.cujae.edu.cu
}

Received: 02 August 2020, Accepted: 26 November 2020, Published online: 19 July 2021

\begin{abstract}
Recently a novel method for radar detection was conceived to process the scattered signal parameters and detect through its statistical moments. Among the advantages of detection in the moments space stands the opportunity of considering the moments like Gaussian random variables, decreasing the uncertainty about the distribution of the variables used by traditional methods. Therefore, it is very important to study the conditions for assuming the above within certain level of confidence. This work uses real radar signals in order to study the influence of two essential variables for detection in the moments space: the sampling interval and the size of the random sample. Average correlation coefficient, hypothesis testing and numerical goodness-of-fit coefficients are used to estimate the values of the previous variables that allow to take the joint distribution of moments as close to the multivariate Gaussian. The guidelines presented should be taken into account for the proper configuration of detectors in the moments space.
\end{abstract}

\section{Keywords}

radar detection, moments space, hypothesis testing, Gaussian distribution

\section{Introduction}

Radar processors require a priori knowledge of the interference distribution in order to establish the threshold that guarantees the design probability of false alarm. The variability of interferences like marine clutter makes it difficult to properly model the diversity of distributions presented in practice [1]. The estimation of the distribution that best describes the parameters of the received signal is an open issue [2-5], with a high complexity associated. The lack of fully satisfactory results in this subject has an impact on the detection quality, leading to unacceptable increases in false alarms or target losses.

Besides the lack of knowledge about the statistical model there is the problem of insufficient discrimination ability of the variables used during detection. It is a general criterion that the parameters of the eco-signals should be processed to increase their possibilities [6-8].

In the last two decades progress has been made in a method with the dual purpose of reducing a priori uncertainty about the statistical model and providing features to increase the detection quality. Known by its authors as DRACEC, a Spanish acronym for Radar Detection through the Analysis and Classification of Cellular Emission, it is grounded on [9] and was first presented in [10].

DRACEC accumulates samples of the parameters (amplitude, frequency, polarization, etc.) of the received radar signal and computes its statistical moments (mean, correlation, etc.), so the decision about the existence of a target is made in the moments space. The fundamental objective of the method is to assign the resolution cells that compose the surveillance region to the background or anomaly classes. In this way the background will be those processes that commonly exist in the whole region, like the clutter. On the other hand, the anomaly will correspond to any disturbance of the background by the presence of an extraneous phenomenon, like the targets to be detected.

Among the advantages of detection in the moments space stands the reduction of the a priori uncertainty about the statistical model of the random process, by assuming that the moments distribution is close to the Gaussian. This reasoning is based on the central limit theorem [11] and the consideration that moments result from adding up a large-size set of decorrelated samples. 
The above discussion arises two worthy questions: what sampling interval guarantees that the samples are decorrelated and what would be the random sample size required to obtain Gaussian moments? Works related to detection through moments can be found in [9, 12-17] but none of them has carried out a study with real radar signals to answer the above mentioned questions. Therefore, the objective of this research is to select suitable values for the sampling interval and the size of random sample to guarantee that the joint distribution of the moments is close to the Gaussian.

The following section deals with the main concepts of detection in the moments space, hypothesis testing and numerical goodness-of-fit coefficients. The selection of suitable values for the size of random sample and sampling interval is shown in the third section, while the fourth verify that using these values, the moments are grouped in elliptical clusters as expected for Gaussian random variables. Finally, the conclusions are presented.

\section{Theoretical fundamentals}

The conceptual framework proposed by DRACEC [9] was the first to consider radar detection with decision making in the moments space. The application of this technique to a general set of signal parameters and three-dimensional surveillance regions is detailed in previous work [9, 14]. In order to facilitate the analysis and notation the method is henceforth particularized to the case of a single parameter: the amplitude of the received signal, which is the more relevant for most applications.

DRACEC divides the surveillance region into a number of resolution cells corresponding to various angular sectors and ranges [14]. A change in any cell will cause disturbances that affect the statistical moments of the underlying random process and therefore can be detected. For each cell $N$ values are computed for each moment from a random sample of size $M$ through [14]

$$
\left[\xi_{k_{1}, \ldots, k_{g}}\right]_{n}=\frac{1}{M} \sum_{m=1}^{M}\left[x_{1}^{k_{1}} \cdots x_{g}^{k_{g}}\right]_{m} \quad n=1, \ldots, N
$$

where $\left[x_{1}^{k_{1}} \cdots x_{g}^{k_{g}}\right]$ constitutes a sample-element formed by the samples of the amplitude $x_{1}, \ldots, x_{g}$ raised to the corresponding magnitude. The sum $k_{1}+k_{2}+\ldots k_{g}$ represents the order of the moment and $g=1, \ldots, G$ is the statistic order, linked in this context to the number of time intervals analyzed [11].

Note the difference between the meanings of sample, sample-element, and random sample. Each term in the summation of Eq. (1) constitutes a sample-element composed by the samples of the random process grouped, multiplied and raised to the corresponding magnitude. The random sample is the whole set of $M$ sample-elements that contribute to the moment computation. The samples might have any degree of correlation but the intervals between sample-elements should ensure decorrelation between them. Therefore, the index $n$ should vary with respect to $m$ so that no consecutive sample-elements are included in the summation until the interval ensuring decorrelation has elapsed. Following this procedure, the distribution of the $N$ moments might approach the Gaussian behavior.

Each resolution cell will be characterized by a set $\Phi$ formed by the $N$ vectors (or patterns) whose components (or features) are the $L$ selected moments. For the purposes of this research $L=3$, so the patterns will be tridimensional vectors denoted as $\boldsymbol{\mu}$ and composed by the well-known moments: mean, mean power and correlation. According to the notation of Eq. (1) these would be the first-order moment of first-order statistic $\left(\xi_{1}, k_{1}=1, g=1\right)$, the second-order moment of first-order statistic $\left(\xi_{2}, k_{1}=2, g=1\right)$ and the second-order moment of second-order statistic $\left(\xi_{11}, k_{1}=1, k_{2}=1, g=2\right)$.

The goal of DRACEC is to use the information contained in $\Phi$ to assign each resolution cell to the classes background or anomaly, hence the link of the acronym with the classification of the cellular emission. The background is associated with the processes that take place "normally" in the searching window, for example, the clutter. Likewise, phenomena outside the "normality" will be treated as anomalies, which constitute the targets to be detected.

The Neyman-Pearson criterion [14] is used to obtain the optimal decision rule, which maximize the probability of detection for a constant probability of false alarm. Therefore, the problem lies in choosing between two hypotheses:

1. the cell belongs to the background class and

2. the cell belongs to the anomaly class.

If the moments are computed from different groups of random samples, it can be assumed that they are independent, and their Joint Probability Density Function (JPDF) is the multiplication of the particular gaussian densities $[11,14]$. However, this requires to compute only one moment for each random sample and increases the complexity of the acquisition algorithm.

On the other hand, if all the moments are computed from the same group of samples like in this paper, they are said to be correlated and its JPDF takes the form [11, 12] 
$p_{C}(\mu)=\frac{\exp \left\{-\frac{1}{2}\left(\mu-\bar{\mu}_{C}\right) \Sigma_{C}^{-1}\left(\mu-\bar{\mu}_{C}\right)^{T}\right\}}{(2 \pi)^{L / 2}\left|\Sigma_{C}\right|^{1 / 2}}$,

where $\boldsymbol{\mu}$ represents the pattern as a row vector, $\overline{\boldsymbol{\mu}}_{C}$ is the mean vector, $\Sigma_{C}$ is the covariance matrix of the moments, while $\left|\Sigma_{C}\right|$ and $\Sigma_{C}^{-1}$ are its determinant and inverse matrix respectively. $T$ denotes the transposed and $C=B, A$ for the background and the anomaly respectively.

From the statistical theory it is well known that Eq. (2) will be valid if and only if the sum of the moments

$[S]_{n}=\left[\xi_{1}\right]_{n}+\left[\xi_{2}\right]_{n}+\left[\xi_{11}\right]_{n} \quad n=1, \ldots, N$,

can be considered Gaussian [11]. The new random variable "sum", denoted as $S$, will be used as a test to establish whether the JPDF of the moments is multivariate Gaussian. The formation of the sample-elements in Eq. (1) through the process of radar signal acquisition [16] directly affects the computation of the moments and $S$. Hence the interest in examining the influence of the sampling interval $\left(T_{m}\right)$ and size of random sample $(M)$ on the Gaussian behavior of $S$.

\subsection{Correlation coefficient}

The correlation coefficient [11] will be used to analyze the sampling interval, which offers a measure of the linear dependence between $x_{1}$ and $x_{2}$ according to

$\rho=\frac{\operatorname{cov}\left(x_{1}, x_{2}\right)}{\sigma_{x_{1}} \sigma_{x_{2}}}$.

The term $\operatorname{cov}\left(x_{1}, x_{2}\right)$ is the covariance between $x_{1}$ and $x_{2}$, which are samples of the received signal separated by $T_{m}$ time units, while $\sigma_{x_{1}}$ and $\sigma_{x_{2}}$ are the standard deviations of the same variables. The correlation coefficient takes values between 1 and -1 , being zero when $x_{1}$ and $x_{2}$ are decorrelated.

According to Nathanson's criterion [18] $\rho$ must be modularly lower than 0.02 for the samples to be decorrelated. The time interval for achieving this can be from milliseconds to seconds [18-20] without agreement about the best alternative. Some values are between $10 \mathrm{~ms}$ and $20 \mathrm{~ms}[18,21,22]$ but this depends on the particular experiment. The values obtained in previous works have a limited utility because none of them analyses the sampling interval for which the moments present a Gaussian JPDF. Therefore, in addition to the correlation coefficient other tools should be used such as hypothesis tests and numerical goodness-of-fit coefficients.

\subsection{Hypothesis tests and numerical goodness-of-fit coefficients}

Hypothesis testing is used to determine whether to reject or accept the null hypothesis $[4,23]$ denoted by $H_{0}$, which in this work will be the hypothesis that $N$ values of the random variable $S$ comes from a population with Gaussian Cumulative Distribution Function (CDF). To reject or accept $H_{0}$ a test statistics is used, which evaluates some characteristic of the samples with respect to its population with a significance level $(\alpha)$ of making a type $I$ error (rejecting $H_{0}$ when it is true) [11]. Typical values for $\alpha$ are between 0.01 and 0.1. Another parameter of interest for hypothesis testing is the $p$-value $[5,24]$, when it is small there is doubt about the truthfulness of the null hypothesis.

Some hypothesis tests are Chi-Square [25], Lilliefors [26], Jarque-Bera [27], Anderson-Darling [2, 28] and Kolmogorov-Smirnov [5, 29-32]. The latter computes the statistic [25]

$d=\max _{S}|\hat{F}(S)-F(S)|$

which is the maximum absolute difference between the empirical CDF $\hat{F}(S)$ of the observed samples and the hypothesized CDF $F(S)$. This test is one of the most classical and has been widely used in the radar field [29-32], therefore will be used hereafter. It also has the advantage of being useful for both large and small sample sizes.

Another way to check that the JPDF of the moments approaches the multivariate Gaussian is through curve fitting [33, 34]. These goodness-of-fit methods also need to know the empirical and hypothesized CDF of the samples to compute numerical coefficients, which evaluate the quality of the fit. Some of these coefficients are the mean square error [35]

MSE $=\frac{1}{N} \sum_{n=1}^{N}\left|\hat{F}\left(S_{n}\right)-F\left(S_{n}\right)\right|^{2}$,

and the normalized mean square error [24]

$\mathrm{NMSE}=1-\frac{\sum_{n=1}^{N}\left[\hat{F}\left(S_{n}\right)-F\left(S_{n}\right)\right]^{2}}{\sum_{n=1}^{N}\left[F\left(S_{n}\right)-\overline{F\left(S_{n}\right)}\right]^{2}}$,

where $\overline{F\left(S_{n}\right)}$ is the mean value of the hypothesized CDF. Values close to 1 for the NMSE and close to 0 for the MSE indicate an ideal fit. 


\subsection{Computation of the moments}

The moments are computed by adding the new acquired sample and discarding the first one from the previous iteration. This is an alternative to Eq. (1) for calculating the accumulated sum and is a moving average stated through the difference equation [36]

$$
y[m]=y[m-1]+\frac{1}{M}(x[m]-x[m-M]),
$$

where $y[m]$ represents the output for the current sample and is also the value of the moment for the previous $M$ samples, $y[m-1]$ is the previous moment value, $x[m]$ is the current sample-element and $x[m-M]$ corresponds to the $M$-th sample-element.

\section{Experiments and results}

The Kolmogorov-Smirnov test and the numerical goodness-of-fit coefficients will be applied to the samples contained in the IPIX database. These signals were acquired in February 1998 with the IPIX Radar [32, 37] from McMaster University of Canada, located in Grimsbay and overlooking Lake Ontario. The system is a coherent experimental radar that works in the X Band, with double polarization for transmission-reception: linear horizontal $(\mathrm{H})$ or vertical $(\mathrm{V})$. In this work the signals received by the in-phase and quadrature channels are demodulated through a quadratic detector [19].

The surveillance region corresponds to the lake surface without a target present and it is divided into 34 range cells, so the acquired signal only resembles the marine clutter. Each cell has 60000 samples equivalent to 1 minute of acquisition and the interval between samples is 1 millisecond, equal to the pulse repetition period $(1 \mathrm{kHz}$ repetition frequency). The database is divided into three datasets identified by the numbers 84,85 and 86 , with resolutions of $30 \mathrm{~m}, 15 \mathrm{~m}$ and $3 \mathrm{~m}$ respectively, each with the four polarizations $\mathrm{HH}, \mathrm{HV}, \mathrm{VH}$ and VV.

\subsection{Selection of the sampling interval}

The selection of the sampling interval is based on the correlation coefficient of Eq. (4), taking $x_{1}$ and $x_{2}$ as the samples of the signal separated by $T_{m}$ milliseconds. Figs. 1-3 show the average correlation coefficient $(\rho)$ for the 34 cells and the four polarizations of each dataset.

Figs. 1-3 show that the correlation coefficient is lower for cross-polarizations and decreases as the radar resolution and sampling interval increases. The condition $\rho \leq 0.5$ is met from 50 milliseconds for the dataset with

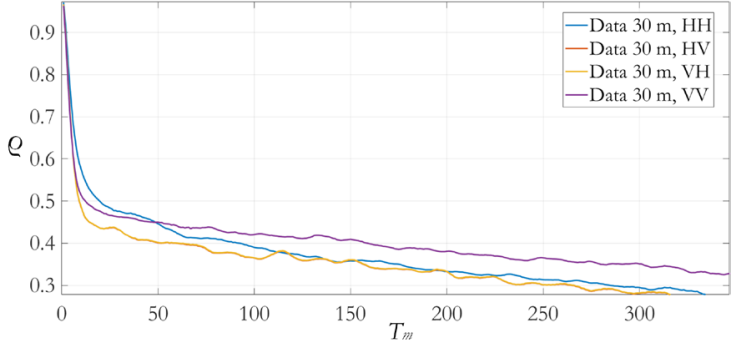

Fig. 1 Average correlation coefficient for dataset with resolution of $30 \mathrm{~m}$

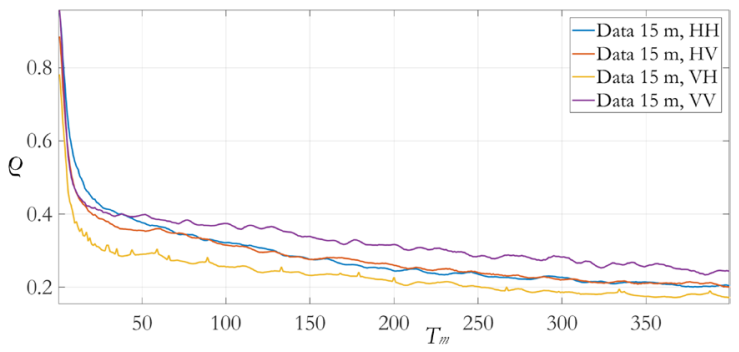

Fig. 2 Average correlation coefficient for dataset with resolution of $15 \mathrm{~m}$

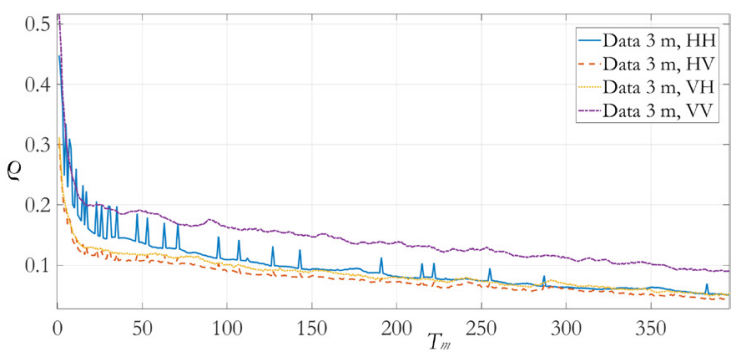

Fig. 3 Average correlation coefficient for dataset with resolution of $3 \mathrm{~m}$

the critical resolution of $30 \mathrm{~m}$. Here the lowest resolution is referred as critical since it takes the largest decorrelation time. Therefore, if a specific requirement is satisfied for the $30 \mathrm{~m}$ dataset it will be fulfilled more easily for the two others datasets.

In order to have some safety margin in terms of the decorrelation time, $T_{m}=100$ milliseconds is taken as sampling interval for all datasets. This choice also tries to keep the same number of computed patterns and hypothesis tests for all resolutions.

\subsection{Selection of the size of random sample}

To select the size of random sample, the rejection percentage of the null hypothesis (that the variable $S$ has a Gaussian CDF) is analyzed for several values of $M$. The sampling interval $T_{m}$ designated in the previous section is taken in to account. The MATLAB function applying the Kolmogorov-Smirnov test is kstest with $\alpha=0.05$, since it is a typical value [24]. This function returns the rejection ("1") or acceptance ("0") of the null hypothesis and the $p$-value. 
The chosen moments were calculated as well as the random variable $S$, which is applied to the test. Figs. 4-6 plot the results for all polarizations of each dataset. It can be seen that the percent of rejection and the $p$-value preserves a similar behavior for all resolutions.

As expected, the rejection rate decreases as $M$ increases since the approximation of Gaussian moments should be more accurate. However, increasing $M$ beyond 60 has generally little impact or even negative effects for some polarizations. The most suitable values for $M$ are around the knee of the curves, therefore $M$ between 10 and 30 would be appropriate, depending on the resolution.

The analysis of the numerical goodness-of-fit coefficients is also performed for $T_{m}=100$ milliseconds. The MATLAB function used for this is goodnessOfFit
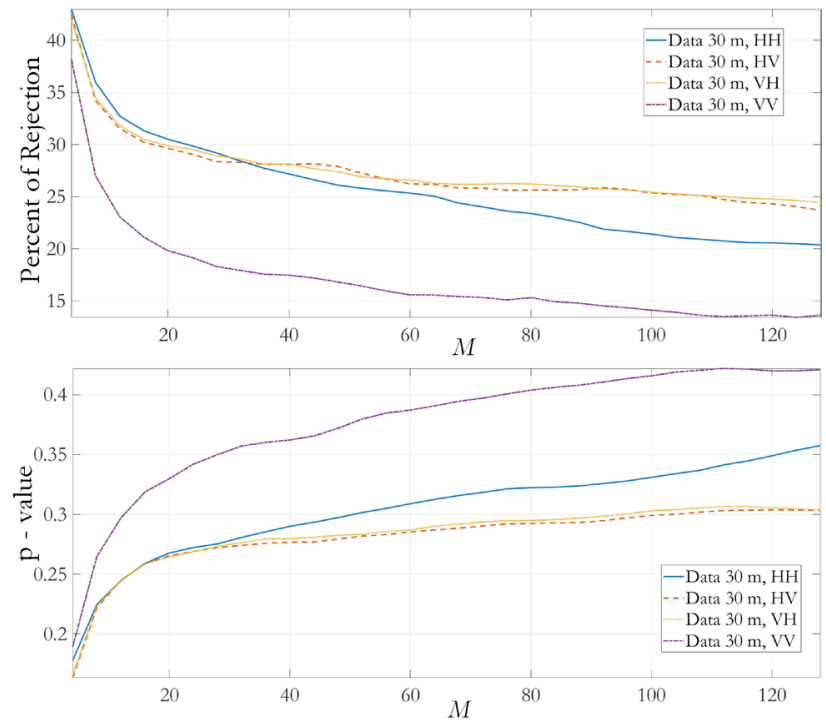

Fig. 4 Percent of rejection and $p$-value for dataset with resolution of $30 \mathrm{~m}$
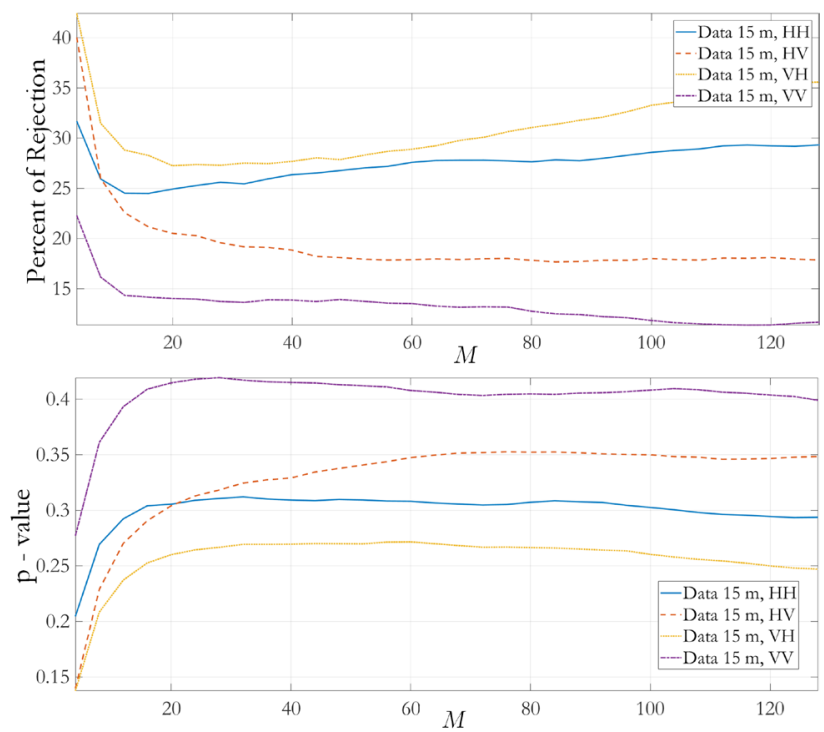

Fig. 5 Percent of rejection and $p$-value for dataset with resolution of $15 \mathrm{~m}$
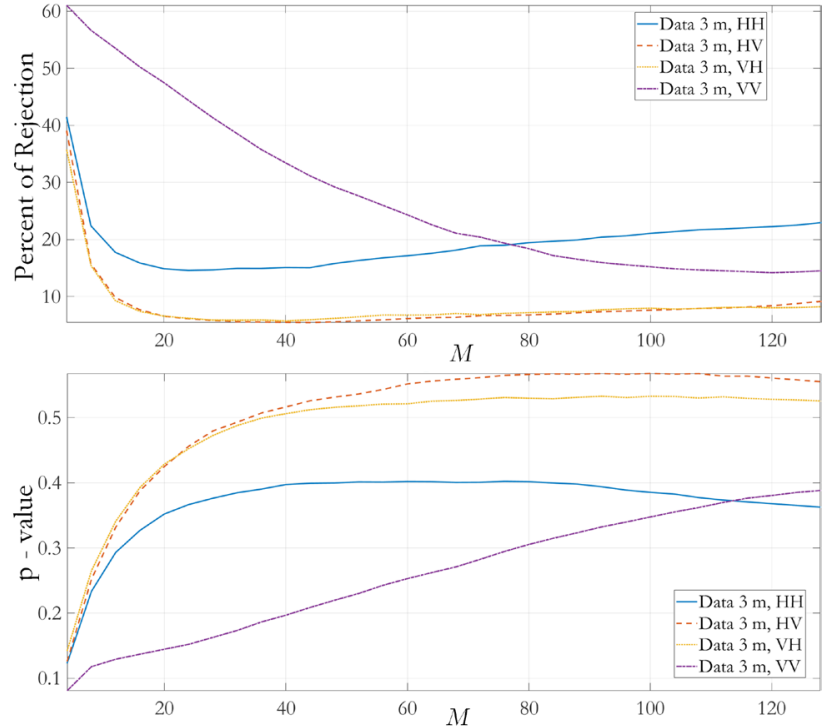

Fig. 6 Percent of rejection and $p$-value for dataset with resolution of $3 \mathrm{~m}$

[24], which returns the MSE and NMSE. The values of these coefficients for all polarizations and resolutions are plotted in Figs. 7-9.

Figs. 7-9 show that as $M$ increases, the MSE approaches zero and NMSE approaches unity, which indicates a better fit to the Gaussian CDF. As in the previous section, increasing $M$ above 60 does not bring noticeable benefits to the numerical coefficients. Again, any value of $M$ between 10 and 30 could be chosen with similar results, therefore $M=20$ could be selected.

\section{Clusters shape in the moments space}

The goal of this section is to illustrate that patterns computed from the values of $M=20$ and $T_{m}=100$ milliseconds resulting from previous analyses are grouped in elliptical
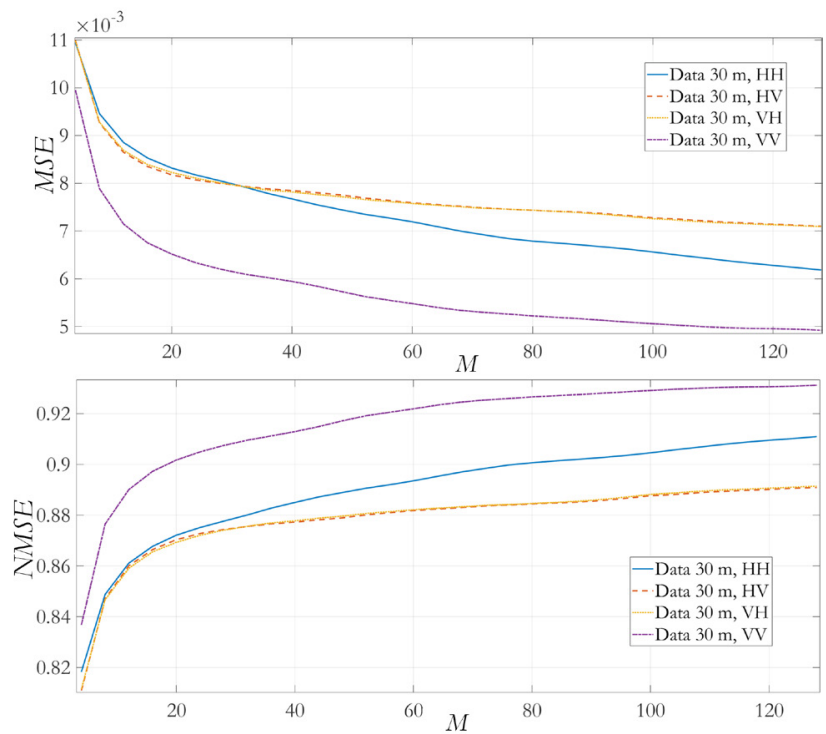

Fig. 7 Goodness-of-fit coefficients for dataset with resolution of $30 \mathrm{~m}$ 

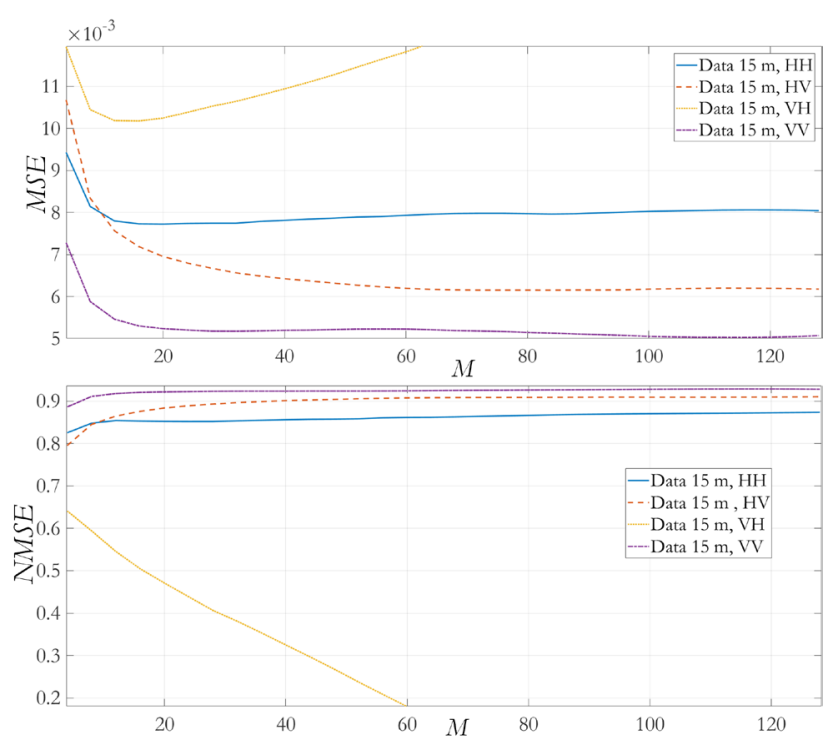

Fig. 8 Goodness-of-fit coefficients for dataset with resolution of $15 \mathrm{~m}$

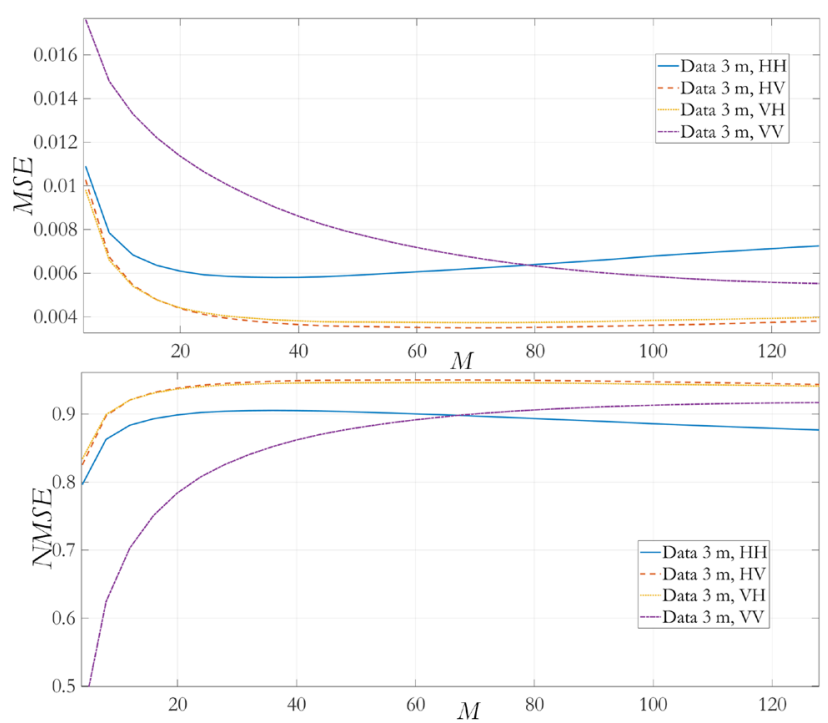

Fig. 9 Goodness-of-fit coefficients for dataset with resolution of $3 \mathrm{~m}$

clusters. This behavior is the expected for moments that follow a multivariate Gaussian JPDF.

Two nearby cells are selected for each resolution and in one of them an anomaly is generated, which follows the Swerling 2 model of fast fluctuation [19, 38]. Results for all polarizations are very similar, so it is only illustrated the HH case. This statement also holds for the others Swerling models. Table 1 shows the cells taken as background and anomaly for each resolution as well as the Signal-toClutter Ratio (SCR) of the synthetic anomaly.

Table 1 Cells and SCR used for the example

\begin{tabular}{lccc}
\hline Dataset Resolution & $30 \mathrm{~m}$ & $15 \mathrm{~m}$ & $3 \mathrm{~m}$ \\
\hline Background Cell & 20 & 7 & 14 \\
Anomaly Cell & 21 & 8 & 15 \\
SCR & $-3 \mathrm{~dB}$ & $0 \mathrm{~dB}$ & $-3 \mathrm{~dB}$ \\
\hline
\end{tabular}

Figs. 10-12 show that the assumption of a multivariate Gaussian JPDF for the moments is satisfactory for the chosen $M$ and $T_{m}$. The elliptical shape of the clusters clearly shows that the JPDF of the moments is multivariate Gaussian. The blue color was used to represent the background patterns, while the red ones corresponds to the anomaly.

Additionally, the figures show that it is possible to differentiate both classes, so the opportunity for detection in the moments space is verified. Note from Table 1 that the SCRs of the anomalies are low, however their patterns are substantially apart from those of the background. This fact manifests the detection potential of the moments space, which is demonstrated in previous works $[14,17]$.

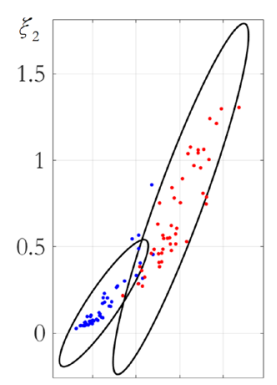

$\begin{array}{lllll}0.2 & 0.4 & 0.6 & 0.8 & \xi_{1}\end{array}$

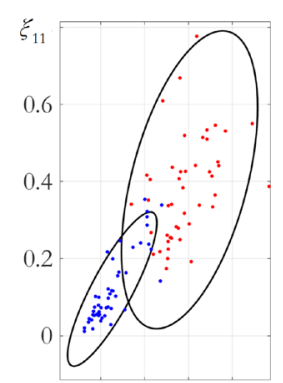

$\begin{array}{lllll}0.2 & 0.4 & 0.6 & 0.8 & \xi\end{array}$

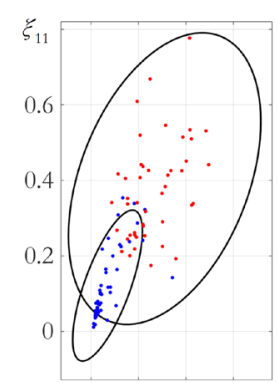

$\begin{array}{lllll}0 & 0.5 & 1 & 1.5 & \xi_{2}\end{array}$
Fig. 10 Moments space for resolution $30 \mathrm{~m}$. Blue patterns corresponds to the background and red ones to the anomaly
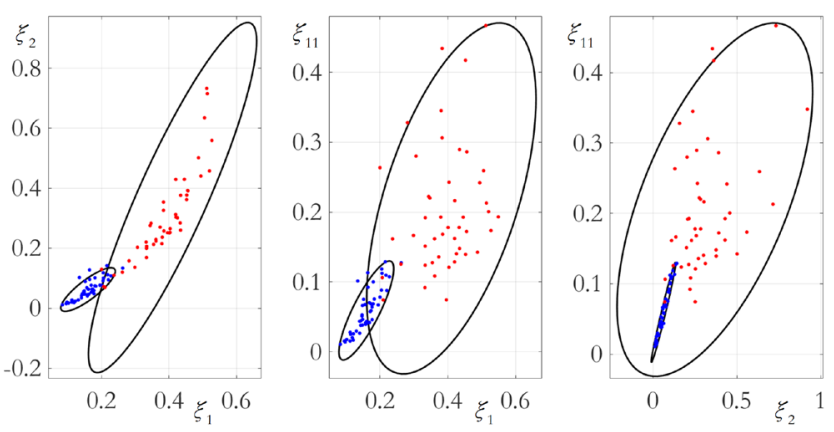

Fig. 11 Moments space for resolution $15 \mathrm{~m}$. Blue patterns corresponds to the background and red ones to the anomaly
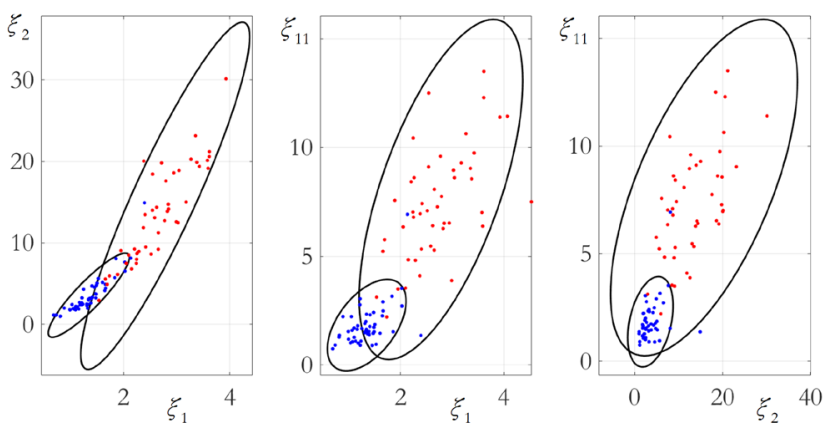

Fig. 12 Moments space for resolution $3 \mathrm{~m}$. Blue patterns corresponds to the background and red ones to the anomaly 


\section{Conclusion}

The influence of the size of the random sample and sampling interval on the condition of Gaussian moments were considered. The values chosen for these two design variables allow proper operation for all available resolutions and polarizations.

In case it is required to operate with specific resolution and polarization, the curves obtained facilitate the selection for the particular case of interest. Furthermore, the

\section{References}

[1] Machado Fernández, J. R., Bacallao Vidal, J. C. "Distribuciones estadísticas para modelar clutter marino: una revisión" (Statistical Distributions for Modeling Marine Clutter: a Revision), Revista de Ingeniería Electrónica, Automática y Comunicaciones, RIELAC, 38(2), pp. 12-35, 2017. [online] Available at: http://scielo.sld.cu/ scielo.php?script $=$ sci_arttext\&pid $=$ S1815-59282017000200002 [Accessed: 31 July 2020] (in Spanish)

[2] Semenova, M. A., Khalin, D. S. "Research of Statistic Distributions of Nonparametric Goodness-of-Fit Test by Large Samples", In: 2018 XIV International Scientific-Technical Conference on Actual problems of Electronics Instrument Engineering (APEIE), Novosibirsk, Russia, 2018, pp. 260-264. https://doi.org/10.1109/APEIE.2018.8545909

[3] Zhou, W., Xie, J., Zhang, B., Li, G. "Maximum Likelihood Detector in Gamma-Distributed Sea Clutter", IEEE Geoscience and Remote Sensing Letters, 15(11), pp. 1705-1709, 2018. https://doi.org/10.1109/LGRS.2018.2859785

[4] Chen, Y., Wu, Y., Chen, N., Feng, W., Zhang, J. "New Approximate Distributions for the Generalized Likelihood Ratio Test Detection in Passive Radar", IEEE Signal Processing Letters, 26(5), pp. 685-689, 2019.

https://oi.org/10.1109/LSP.2019.2903632

[5] Hasanshahi, Z., Azmi, P., Gholizadeh, M. H., Khajezadeh, M. "The Flexibility of the Generalized Gamma Distribution in Modeling the Fading Based on Kullback-Leibler and KolmogorovSmirnov Criteria", IEEE Access, 8, pp. 8393-8404, 2020. https://doi.org/10.1109/ACCESS.2020.2964561

[6] Shi, S.-N., Shui, P.-L. "Sea-Surface Floating Small Target Detection by One-Class Classifier in Time-Frequency Feature Space", IEEE Transactions on Geoscience and Remote Sensing, 56(11), pp. 6395-6411, 2018.

https://doi.org/10.1109/TGRS.2018.2838260

[7] Guo, Z., Shui, P. "Sea-surface floating small target detection based on feature compression", The Journal of Engineering, 2019(21), pp. 8160-8164, 2019.

https://doi.org/10.1049/joe.2019.0694

[8] Shi, S. N., Liang, X., Shui, P. L., Zhang, J. K., Zhang, S. "Low-Velocity Small Target Detection with Doppler-Guided Retrospective Filter in High-Resolution Radar at Fast Scan Mode", IEEE Transactions on Geoscience and Remote Sensing, 57(11), pp. 8937-8953, 2019.

https://doi.org/10.1109/TGRS.2019.2923790 validity of the described tests exceeds any concrete example since they could be applied in a general way to any other scenario. The guidelines presented should be taken into account for the design of radar detectors that intend to exploit the advantages of the moments space.

\section{Acknowledgement}

Authors want to thank McMaster University for making the IPIX database freely available.

[9] Chávez, N. "Detección y Alcance de Radar: La Alerta Temprana de Blancos en Fondos Enmascarantes y una Solución al Problema" (Radar Detection and Ranging: Early Warning of Targets in Masking Backgrounds and a Solution to the Problem), PhD Thesis, Instituto Técnico Militar "José Martí", 2002. (in Spanish) https://doi.org/10.13140/RG.2.2.35406.97606

[10] Chávez, N., González, A. L. "Radar Recognition through Statistical Classification of Cellular Emission in the Moment Space", In: Ruiz-Shulcloper, J., Kropatsch, W. G. (eds.) Progress in Pattern Recognition, Image Analysis and Applications, Springer, Berlin, Heidelberg, Germany, 2008, pp. 325-331.

https://doi.org/10.1007/978-3-540-85920-8_40

[11] Papoulis, A. "Probability, Random Variables, and Stochastic Processes", McGraw-Hill, New York, USA, 1991.

[12] Guillén Soriano, C., Chávez Ferry, N. "Densidad de Probabilidad Conjunta Normal Multivariada para Momentos Correlacionados" (Multivariate Normal Joint Probability Density for Correlated Moments), In: XVIII Convención Científica de Ingeniería y Arquitectura, La Habana, Cuba, 2016, pp. 1-6. [online] Available at: https://www.researchgate.net/publication/309673079_ MULTIVARIATE_NORMAL_PROBABILITY_DENSITY FUNCTION_FOR_CORRELATED_MOMENTS [Accessed: 31 July 2020] (in Spanish)

[13] Guillén Soriano, C., Chávez Ferry, N. "Detección por Radar en el Espacio de los Momentos: Análisis de un Caso Particular" (Radar Detection in the Moments Space: Analysis of a Particular Case), In: VII Simposio Internacional de Telecomunicaciones, Havana, Cuba, 2016. [online] Available at: https://www.researchgate.net/ publication/300156657_Deteccion_por_Radar_en_el_Espacio_ de_los_Momentos_Analisis_de_un_Caso_Particular [Accessed: 31 July 2020] (in Spanish)

[14] Chávez, N., Guillén, C. "Radar Detection in the Moments Space of the Scattered Signal Parameters", Digital Signal Processing, 83, pp. 359-366, 2018.

https://doi.org/10.1016/j.dsp.2018.08.013

[15] Guillén Soriano, C. "Formación de la Muestra Aleatoria y Cálculo de los Momentos Estadísticos para un Detector DRACEC" (Formation of the Random-Sample and Computation of the Statistical Moments for a DRACEC Detector), MSc Thesis, Universidad Tecnológica de La Habana "José Antonio Echeverría", CUJAE, 2018. (in Spanish) https://doi.org/10.13140/RG.2.2.10577.17765 
[16] Guillén, C., Casas, G., Frómeta, D., Chávez, N. "Simple Communication Interface for a Radar Detector in the Moments Space", International Journal of Engineering and Technology Innovation, 9(4), pp. 314-326, 2019. [online] Available at: http://ojs.imeti.org/index.php/IJETI/article/view/3815 [Accessed: 31 July 2020]

[17] Guillén, C., Chávez, N. "Two-Dimensional Determination of the Decision Boundary for a Radar Detection Method in the Moment Space", Journal of Aerospace Technology and Management, 11, Article number: e2219, 2019.

https://doi.org/10.5028/jatm.v11.1034

[18] Nathanson, F. E., Reilly, J. P., Cohen, M. N. "Radar Design Principles", McGraw-Hill, New York, USA, 1991. [online] Available at: https://selasl.files.wordpress.com/2011/08/mcgraw hill__radar_design_principles.pdf\&ved=2ahUKEwja1e2Uy vjqAhVrAp0JHbFBDecQFjAHegQIBBAB\&usg=AOvVa w3WGQQUFJ7_0mFlwDjlnMtS [Accessed: 31 July 2020]

[19] Richards, M. A. "Fundamentals of Radar Signal Processing", McGraw-Hill Education, Chicago, USA, 2014. [online] Available at: https://pdf-drive.com/fundamentals-of-radar-signalprocessing-2nded-0071798323-9780071798327/ [Accessed: 31 July 2020]

[20] De Maio, A., Greco, M. S. "Modern Radar Detection Theory", SciTech Publishing, Edison, NJ, USA, 2016. https://doi.org/10.1049/SBRA509E

[21] Palamà, R., Ritchie, M., Griffiths, H., Miceli, W., Fioranelli, F., Sandenbergh, S., Inggs, M. "Correlation Analysis of Simultaneously Collected Bistatic and Monostatic Sea Clutter", In: 2017 IEEE Radar Conference, Seattle, WA, USA, 2017, pp. 1466-1471. https://doi.org/10.1109/RADAR.2017.7944438

[22] Ward, K., Tough, R., Watts, S. "Sea Clutter Scattering, the K Distribution and Radar Performance", IET, London, UK, 2013. https://doi.org/10.1049/PBRA025E

[23] Vu, V. T., Pettersson, M. I., Gomes, N. R. "Stability in SAR Change Detection Results Using Bivariate Rayleigh Distribution for Statistical Hypothesis Test", In: 2019 IEEE International Geoscience and Remote Sensing Symposium (IGARSS 2019), Yokohama, Japan, 2019, pp. 37-40. https://doi.org/10.1109/IGARSS.2019.8898728

[24] MathWorks "Matlab Help (ver. R2017a)", [computer program] Available at: https://www.mathworks.com/help/matlab/releasenotes-R2017a.html [Accessed: 31 July 2020]

[25] Ren, W., Song, J., Zeng, J., Zhang, X. "A Novel Asymmetrical Probability Density Function for Modeling Log-Ratio SAR Images", IEEE Geoscience and remote sensing letters, 13(3), pp. 369-373, 2016. https://doi.org/10.1109/LGRS.2015.2513820

[26] Amale, V. S., Kolhar, S. U., Shastri, R. K., Das, A. "Non-Gaussianity and non-stationary detection in underwater ambient noise using hypothetical test", In: 2016 Conference on Advances in Signal Processing (CASP), Pune, India, 2016, pp. 270-275. https://doi.org/10.1109/CASP.2016.7746178

[27] Fang, Y., Min, H., Wang, W., Xu, Z., Zhao, X. "A Fault Detection and Diagnosis System for Autonomous Vehicles Based on Hybrid Approaches", IEEE Sensors Journal, 20(16), pp. 9359-9371, 2020. https://doi.org/10.1109/JSEN.2020.2987841
[28] Han, D., Tan, X., Shi, P. "Clutter distribution identification based on anderson-darling test", In: 2017 3rd IEEE International Conference on Computer and Communications (ICCC), Chengdu, China, 2017, pp. 830-834.

https://doi.org/10.1109/CompComm.2017.8322660

[29] del-Rey-Maestre, N., Jarabo-Amores, M.-P., Mata-Moya, D., Gomez-del-Hoyo, P., Bárcena-Humanes, J.-L. "Statistical analysis of UHF bistatic radar clutter in coastal scenarios", In: 2015 European Radar Conference, Paris, France, 2015, pp. 253-256. https://doi.org/10.1109/EuRAD.2015.7346285

[30] Ambrosanio, M., Baselice, F., Ferraioli, G., Ferrentino, E., Pascazio, V. "Kolmogorov Smirnov test based approach for SAR automatic target recognition", In: IEEE International Geoscience and Remote Sensing Symposium (IGARSS), Fort Worth, TX, USA, 2017, pp. 1660-1663. https://doi.org/10.1109/IGARSS.2017.8127292

[31] Jianbin, L., Shusen, T., Zemin, X. "Frame segmentation and recognition algorithm for ship's HRRPs based on hypothesis testing", In: 2016 CIE International Conference on Radar (RADAR), Guangzhou, China, 2016, pp. 1-4. https://doi.org/10.1109/RADAR.2016.8059572

[32] Li, Z., Zhao, Z., Zhao, Y. "Analysis of Experimental dData of IPIX Radar", In: 2018 IEEE International Conference on Computational Electromagnetics (ICCEM), Chengdu, China, 2018, pp. 1-3. https://doi.org/10.1109/COMPEM.2018.8496687

[33] Kurnaz, Ç., Engiz, B. K. "Distribution fitting for long term electric field strength measurements", In: 2017 40th International Conference on Telecommunications and Signal Processing (TSP), Barcelona, Spain, 2017, pp. 267-270. https://doi.org/10.1109/TSP.2017.8075984

[34] Daries, R. S., Mishra, A. K. "Complex Wavelet Structural Similarity quality measures for compressively sensing SAR images", In: 2014 IEEE Radar Conference, Cincinnati, OH, USA, 2014, pp. 0892-0895. https://doi.org/10.1109/RADAR.2014.6875717

[35] Xin, Z., Liao, G., Yang, Z., Y. Zhang, Dang, H. "Analysis of Distribution Using Graphical Goodness of Fit for Airborne SAR Sea-Clutter Data", IEEE Transactions on Geoscience and Remote Sensing, 55(10), pp. 5719-5728, 2017. https://doi.org/10.1109/TGRS.2017.2712700

[36] Oppenheim, A. V., Schafer, R. W. "Discrete-Time Signal Processing", Pearson Higher Education, Upper Saddle River, NJ, USA, 2010. [online] Available at: https://www.pearson.com/us/ higher-education/program/Oppenheim-Discrete-Time-SignalProcessing-3rd-Edition/PGM212808.html [Accessed: 31 July 2020]

[37] Mata-Moya, D., del-Rey-Maestre, N., Jarabo-Amores, M.-P., Martinde-Nicolas, J., Barcena-Humanes, J.-L. "An adaptive threshold technique for the LR detector in K-clutter. Validation using IPIX radar", In: 2015 IEEE International Instrumentation and Measurement Technology Conference (I2MTC), Pisa, Italy, 2015, pp. 794-799. https://doi.org/10.1109/I2MTC.2015.7151370

[38] Guillén, C., Chávez, N. "Algorithms to Generate Random Samples following the Swerling Models", Revista Cubana de Ciencias Informáticas, 13(2), pp. 1-12, 2019. [online] Available at: https:// www.researchgate.net/publication/335104476_Algorithms_to_ Generate_Random_Samples_following_the_Swerling_Models [Accessed: 31 July 2020] 